\title{
THERMAL PLASMA OF ELECTRIC ARC DISCHARGE BETWEEN COMPOSITE CU-Cr ELECTRODES: OPTICAL EMISSION AND ELECTRODE SURFACE INTERACTION
}

\author{
A. Murmantsev ${ }^{a, *}$, A. Veklich ${ }^{a}, \mathrm{~V}$. Boretskij ${ }^{a}, \mathrm{M}$. Bartlová ${ }^{b}$,

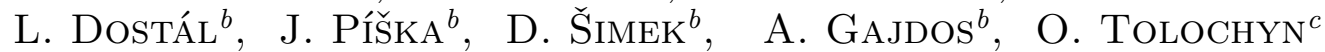 \\ a Taras Shevchenko National University of Kyiv, 64/13, Volodymyrska Street, City of Kyiv, Ukraine, 01601 \\ ${ }^{b}$ Faculty of Electrical Engineering and Communication, Brno University of Technology, Technicka 10, 61600 \\ Brno, Czech Republic \\ ${ }^{c}$ Frantsevich Institute for Problems of Materials Science NAS of Ukraine, 3, Krzhizhanovsky str., Kyiv, Ukraine, \\ 03142 \\ * murmantsev.aleksandr@gmail.com
}

\begin{abstract}
This work deals with investigations of thermal plasma of electric arc discharge between sintered composite $\mathrm{Cu}-\mathrm{Cr}$ electrodes, which can be used in electrical contacts of vacuum circuit breakers. Breaking arcs between composite $\mathrm{Cu}-\mathrm{Cr}$ as well as single-component copper electrodes were used to study the electrical properties, plasma optical emission and electrodes surface modification behavior. In particular, the temporal evolution of plasma emission spectra of electric breaking arcs in air atmosphere was investigated by Optical Emission Spectroscopy (OES). Scanning Electron Microscopy (SEM) with Energy-dispersive X-ray Spectroscopy (EDXS) were applied to analyze the cross-section of working layer of electrodes surface modified by the heat flux from the discharge.
\end{abstract}

Keywords: composite electrodes, spectroscopy, microscopy, arc dicharge, copper, chromium.

\section{Introduction}

Composite materials based on two metals, one of which provides the required electrical conductivity $(\mathrm{Ag}, \mathrm{Cu})$ and the other has the appropriate temperature resistance $(\mathrm{Mo}, \mathrm{W})$ are widely used in various electrical power industry applications. Composites, where chromium is used as a refractory component, are widely used as contact materials in vacuum arc quenching chambers in high and medium load systems $[1,2]$. The erosion resistance of such materials depends on the operating conditions, their formulations and manufacturing methods. The study of the processes taking place during the interaction of composite materials and high temperature sources will contribute to the improvement of production technologies and the optimization of the development of these materials.

At present, the improvement of production technologies and the study of the optimal composition of these materials is still underway.

For example, in a few past decades, several methods of $\mathrm{Cu}-\mathrm{Cr}$ developing, such as, casting [3], arc-melting [4], mechanical or surface alloying $[5,6]$, inducing by electron beam [7] etc, are proposed. Nowadays, the most common method of such composite production is powder metallurgy $[2,8,9]$.

In particular, such composite materials can be produced by sintering and/or infiltration technologies. Small residual porosity can take place (as rule) in $\mathrm{Cu}$ Cr contact materials [2] produced by such methods, which, in turn, can increase the delayed interruption characteristics at high voltages. In some applications, this may affect the breaking ability of the switching device. The electrical and thermal conductivity characteristics can be improved by reduction of the porosity after sintering, for example by pressing [10]. The reduced porosity is advantageous for the breaking capability of the device. In addition, material porosity can be reduced by the temperature increase during sintering [11].

The composite material can be optimized by changing the amount of copper with respect to chromium and find the most appropriate composition to specific applications. As it was found in [12], the erosion resistance of $\mathrm{Cu}-\mathrm{Cr}$ electrodes increases with increasing of chromium content. The component dependence of electrodes ${ }^{6}$ materials wear was studied in [13], in which it was shown that $\mathrm{Cu}-45-50 \% \mathrm{Cr}$ material provides the lowest electrode wear. However, the best interruption rate is provided by $\mathrm{Cu}-25 \% \mathrm{Cr}$ [14], while the increasing of chromium content leads to high number of failures. If it is important for effectiveness of arc quenching at both low and high frequencies at low interrupt current, the required content of chromium is $25 \%$ by the mass $[1,2,15]$. However, if erosion resistance and high durability are important, chromium content of $40-60 \%$ by mass should be used [10].

According to information of the $\mathrm{Cu}-\mathrm{Cr}$ composite electrodes developers e.g. [10], the erosion properties of the aforementioned materials play a key role in the efficiency of switching devices in power grids due to important properties as: 


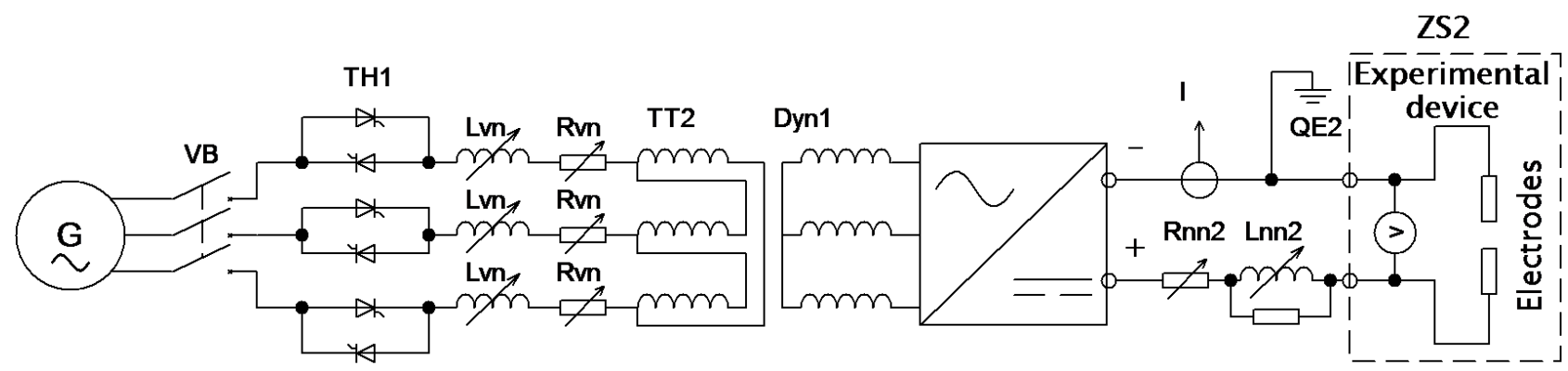

Figure 1. Power circuit and electrical values measurement scheme.

- mechanical strength;

- low gas content;

- perfect resistance to arc erosion;

- high possibility to short-circuit current scattering;

- low penchant to welding;

- breaking ability up to $63 \mathrm{kA}$;

- maximal interrupt current $<6 \mathrm{kA}$.

This paper is focused on investigations of plasma optical emission of electric arc between composite $\mathrm{Cu}$ Cr $(35 \% \mathrm{Cu}$ and $65 \% \mathrm{Cr}$ by the mass), pressured at sintered temperature of $750^{\circ} \mathrm{C}$, and single-component $\mathrm{Cu}$ electrodes as well as in studies of the peculiarities of the working surface of the electrodes affected by the thermal action of the discharge plasma. Specifically, the spectral composition of plasma radiation in the optical range and its time evolution are studied by OES. SEM with EDXS are used to investigate the surface structure and elemental analysis of the cross-section of the working layer of $\mathrm{Cu}-\mathrm{Cr}$ and $\mathrm{Cu}$ electrodes modified by the heat flux of a direct current electric discharge.

\section{Peculiarities of experimental study}

Experiments were performed in High current laboratory at Brno University of Technology (BUT), Czech Republic. The main target of the facility is high power experimentation in developing and testing of a wide range of switching devices.

The power circuit and electrical parameters measurement were realised according to scheme in Fig. 1.

Synchronous generator $\mathrm{G}$, protected with a vacuum circuit breaker VB, was used as the DC power source at current up to $5 \mathrm{kA}$. The main switching was performed by thyristors TH1. Values of current and time constant were adjusted using loads (medium voltage side coils Lvn and resistors Rvn and low voltage side coils Lnn2 and resistors Rnn2). The medium voltage of $6 \mathrm{kV}$, produced by the power generator, was reduced to the required level by short-circuit transformer with in series connected six pulse rectifier TT2 and Dyn1. The electrodes assembly was mounted to the ZS2 test bench. Voltage and current were measured by the data acquisition system (DAQ) with a sampling rate of $50 \mathrm{kHz}$.
The experimental device consisted of the upper fixed holder of electrode (cathode) and the another one (moving) holder of electrode (anode), which is controlled by a pneumatic drive, see Fig. 2.

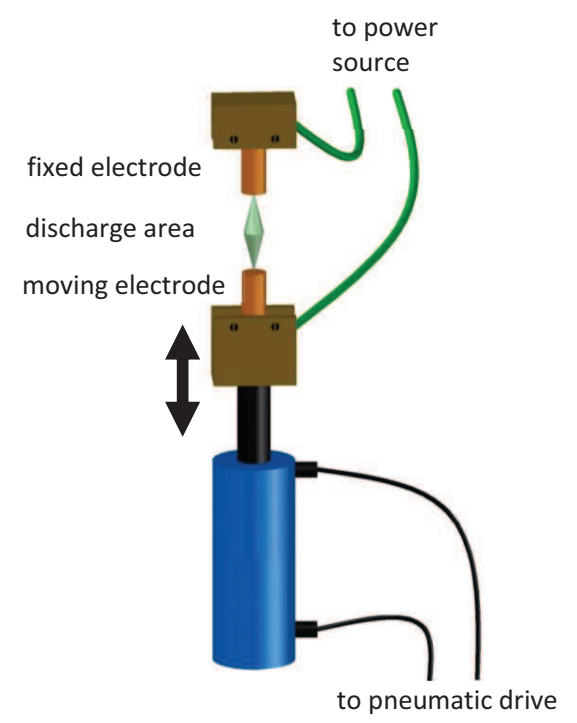

Figure 2. Scheme of experimental device.

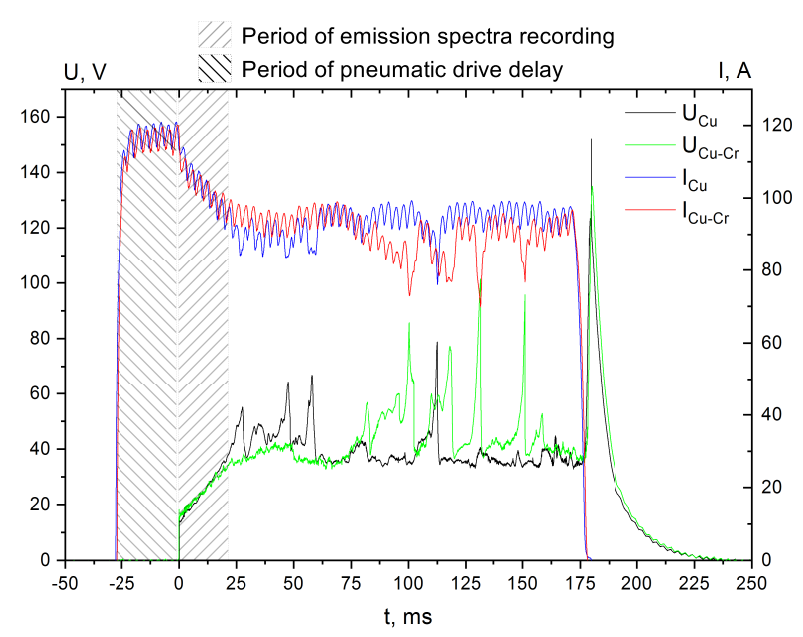

Figure 3. Oscillogram of voltage and current of electric arc discharges between single-component $\mathrm{Cu}$ and composite $\mathrm{Cu}$-Cr electrodes.

Testing procedure description: 

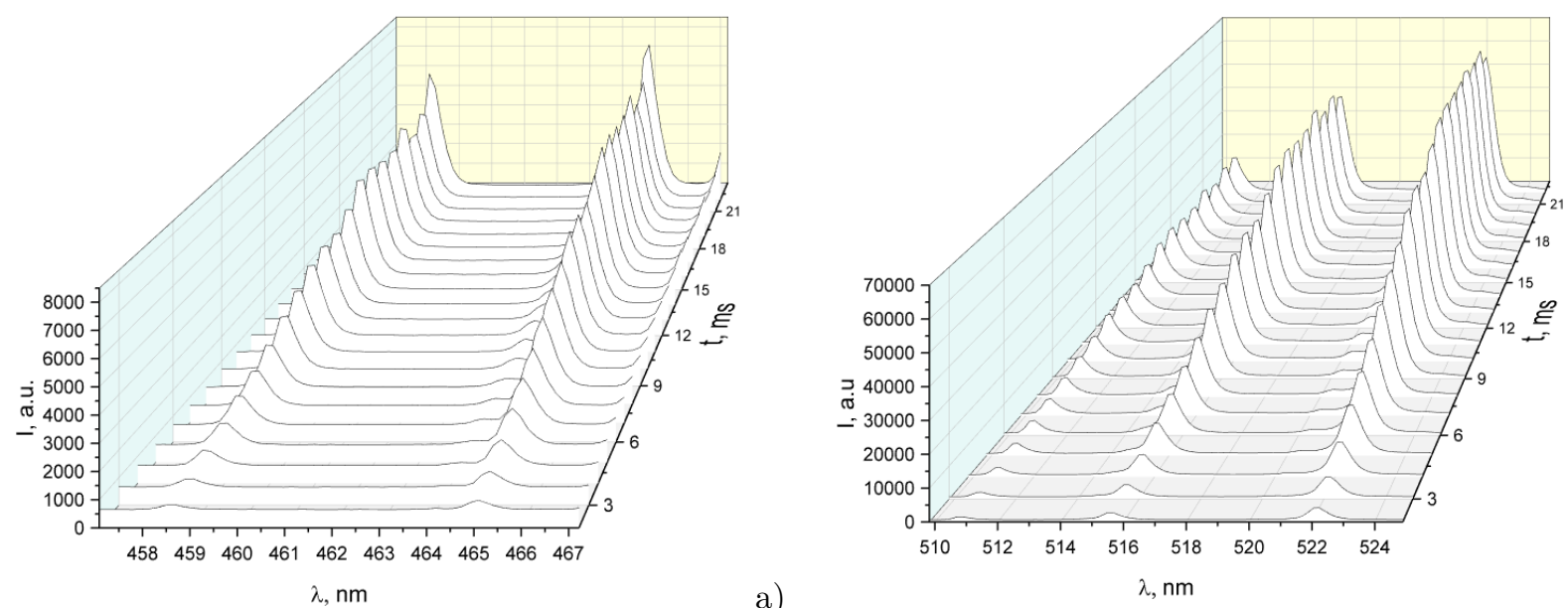

b)
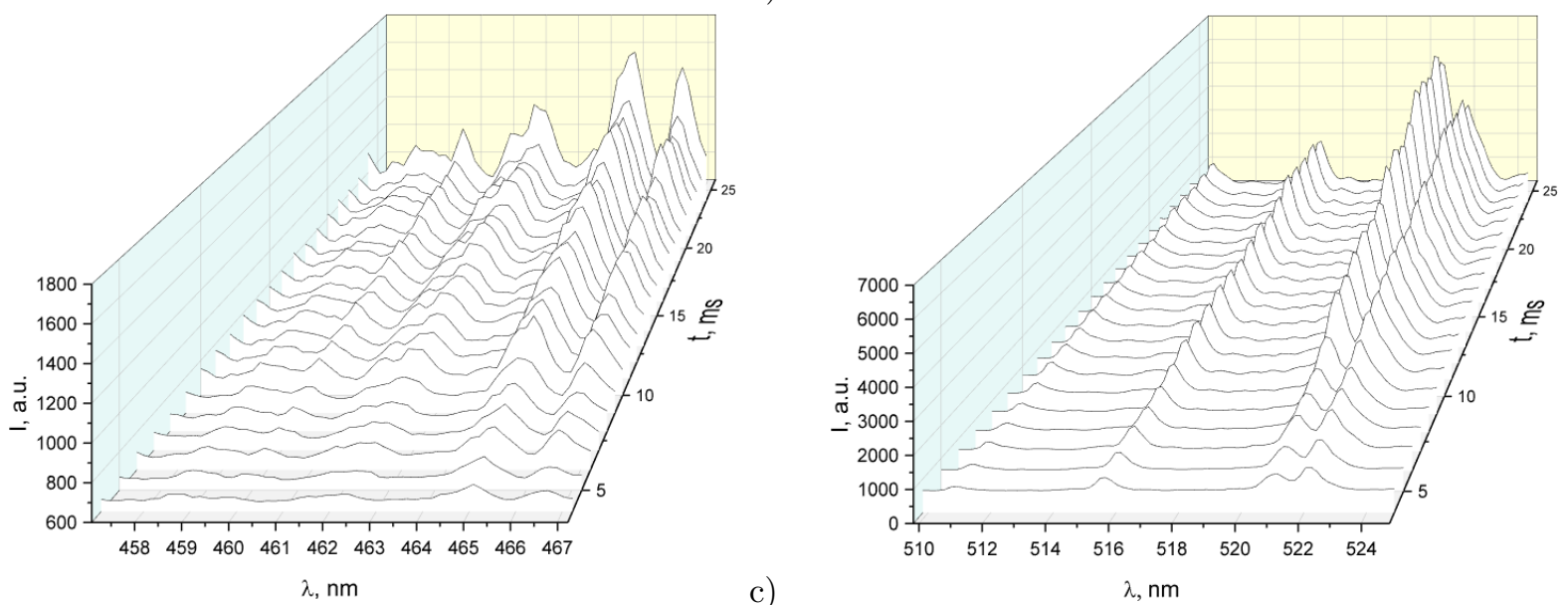

Figure 4. The temporal evolution of arc discharge plasma emission at arc current of 120 A between: a) and b) single-component copper electrodes; c) and d) composite $\mathrm{Cu}$ - $\mathrm{Cr}$ electrodes (spectral range: a) and c) 457-467 $\mathrm{nm}$; b) and d) 510-525 $\mathrm{nm}$ ).

1. Tested electrodes were preliminary weighed (optionally) on the scales (Radwag AS60/220.R2) and put into electrode holders.

2. Electrodes were short-circuited before the beginning of each test.

3. A voltage was switched on by the thyristors and applied between the input and output terminal. A current, limited by loads, was started to flow through the circuit with shorted electrodes.

4. The pneumatic drive was activated after approximately $30 \mathrm{~ms}$, which is the starting point of arc ignition process in the gap between the electrodes.

5. Plasma optical emission spectra were recorded during short initial arcing time period (see Fig. 3).

6. Tested electrodes were removed from the electrode holders and weighed (optionally) on the scales.

As one can see from Fig. 3, the energy, applied to both type of electrodes are the same in the entire period of emission spectra recording. This fact allows to provide a qualitative comparison of emission of electrodes materials under the same conditions.

It must be noted, due to short time of arc combustion, the mass changes were too small for its accuracy measurement. Moreover, the number of experiments was insignificant due to limited quantity of specimens, which did not allow reaching the necessary statistics of the obtained results and obtaining the accurate values of electrode's mass.

The spectrograph Andor Shamrock 500i was used for emission spectra registration. This spectrograph can achieve a high temporal resolution, up to about 10000 spectra per second with fast-kinetics mode. A grating with 150 lines per $\mathrm{mm}$ and blaze of $800 \mathrm{~nm}$ was used and the spectral range was chosen from $400 \mathrm{~nm}$ to $800 \mathrm{~nm}$.

\section{Results and discussions}

The spectra of plasma optical radiation registered in narrow spectral range (457-467 $\mathrm{nm}$ and 510-525 nm) starting from arc ignition are shown for comparison of spectral emission in plasma between electrodes manufactured of different materials (Fig. 4).

As one can see, the emission spectra of plasma between $\mathrm{Cu}-\mathrm{Cr}$ composite electrodes differ from those obtained in plasma between single-component copper electrodes in the same spectral ranges. This difference is caused by the presence of chromium lines in the 
spectrum (see Fig. 4 c, d). In addition, it should be emphasized that the emission intensity (in arbitrary units) of the $\mathrm{Cu}$ I spectral lines for the discharge plasma between the single-component copper electrodes is much higher than between the composite electrodes (see Fig. 5). This may indicate that the concentration of copper vapor in the plasma volume is higher for the discharge between the single-component copper electrodes, and consequently, the composite electrodes have better resistance to erosion caused by the heat flux from the electric arc discharge.

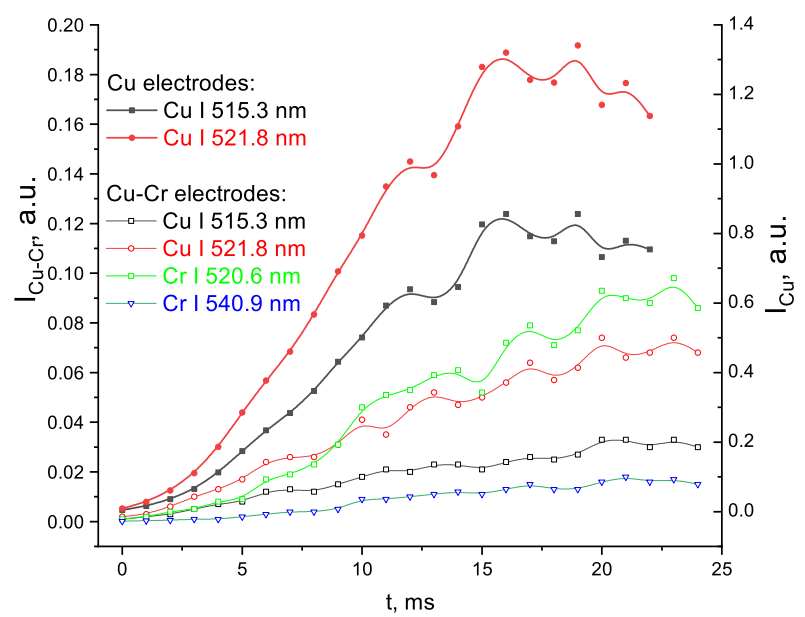

Figure 5. The temporal evolution of radiation intensities of $\mathrm{Cu} I$ 515.3, 521.8 $\mathrm{nm}$ and $\mathrm{Cr} I$ 520.6, $540.9 \mathrm{~nm}$ spectral lines in the plasma between composite $\mathrm{Cu}-\mathrm{Cr}$ electrodes (right axis) and single-component $\mathrm{Cu}$ electrodes (left axis).

In addition, as can be seen in Fig. 5, the time evolution of the emission intensities of each spectral line in the discharge plasma between the single-component copper electrodes achieves a faster saturation compared to the $\mathrm{Cu}-\mathrm{Cr}$ plasma. This phenomenon, once again, confirms the assumption that the $\mathrm{Cu}-\mathrm{Cr}$ electrodes have the better resistance to arc erosion in comparison with $\mathrm{Cu}$ electrodes. The observed oscillations of the emission intensity temporal evolutions can be explained by according character of current oscillation (see Fig. 3).

The metallography study were carried out by SEM with EDXS at the microscope voltage of $20 \mathrm{kV}$ (see Fig. 6-10). The view field and scaling of the pictures were up to $30 \mu \mathrm{m}$ and $5 \mu \mathrm{m}$, respectively (see the legend for a) and b) images in Fig. 6, 8 and 10).

The structure images of non-modified surface of such electrodes are prepared to determine the behaviour of each composite component (Fig. 6 and 7). As one can conclude, the chromium component is predominant in $\mathrm{Cu}-\mathrm{Cr}$ composite material according to fabrication. It can be stressed, that the different components of the composite materials are sharply bordered before treated by arc discharge.

Fig. 8 and 9 show that the structure of composite electrodes is essentially changed after influence of the heat flux from the dicharge. Specifically, depleted copper area is formed in the composite cathode working layer modified by thermal plasma in comparison with non-modified surface (Fig. 7). This phenomenon occurs due to the fact that copper has lower melting temperature than chromium [16]. Due to this fact the copper component of composite electrodes is melted faster than chromium under influence of the heat flux from the discharge.

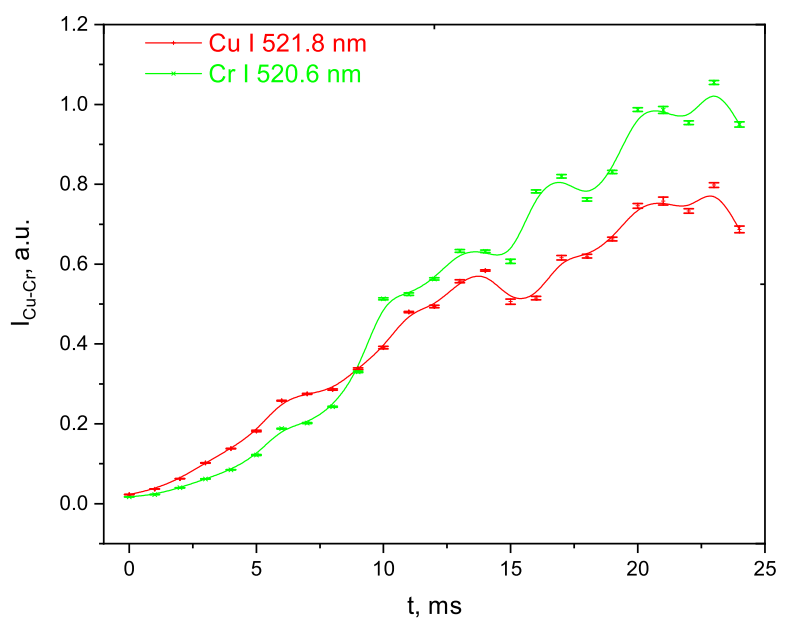

Figure 12. The temporal evolution of radiation intensities of $521.8 \mathrm{~nm}$ and $\mathrm{Cr} I 520.6 \mathrm{~nm}$ spectral lines for the plasma of discharge between composite $\mathrm{Cu}-\mathrm{Cr}$ electrodes.

This effect is shown in Fig. 12. As one can see, the emission intensity of $\mathrm{Cu}$ I spectral line prevails over Cr I spectral line over the time span of $9 \mathrm{~ms}$ and than the intensity of chromium line becomes higher in comparison with that of copper. The reason is that, the emission of copper vapour is higher than chromium vapour in the initial stage of the arcing up to the moment, when copper evaporation from cathode area in working layer and in its vicinity is decreased due to its depletion (Fig. 9 a).

It must be noted, that the presence of gold and carbon on EDXS mapping is to provide the necessary electrical conductivity and to obtain the proper images as a part of technological process of measurements.

Another effect that can be observed in $\mathrm{Cu}-\mathrm{Cr}$ electrodes is copper component behavior in anode. As it was mentioned above, the components of initial structure of composite electrodes have sharp borders in electrodes volume. As one can see from Fig. 8 and 9 , these borders are blurred in the area in vicinity of working layer after modifying by the heat flux from the discharge. It can be stressed, that melting of copper in anode is realised not only in arc spot but in the volume in vicinity of working surface.

This phenomenon is clearly observed in the cathode surface as well. But, as it was mentioned above, copper component is evaporated from the area in vicinity to cathode working surface. The reason is that the arc energy, transferred to the cathode, is higher in 

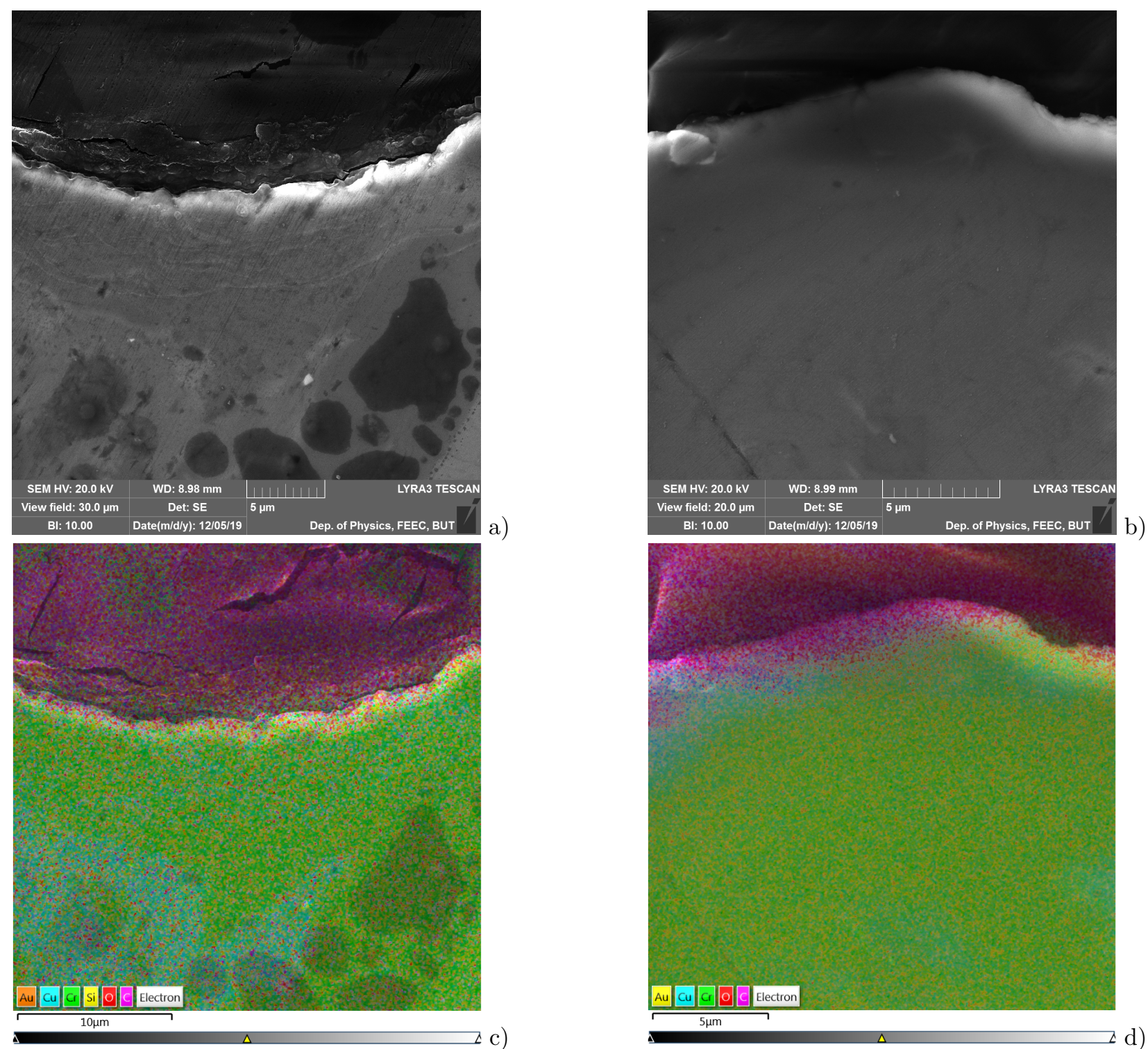

Figure 6. SEM cross-section images ( $a$ and b) and SEM cross-section images with elemental EDXS mappinng analysis ( $c$ and d) of non-modified $\mathrm{Cu}$-Cr composite electrodes layer: a) and c) - cathode surface; b) and d) - anode surface.

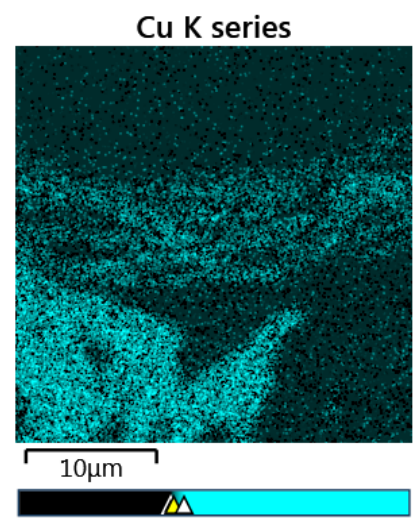

a)

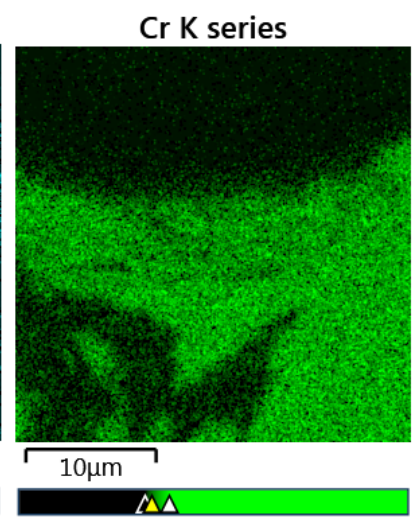

b)

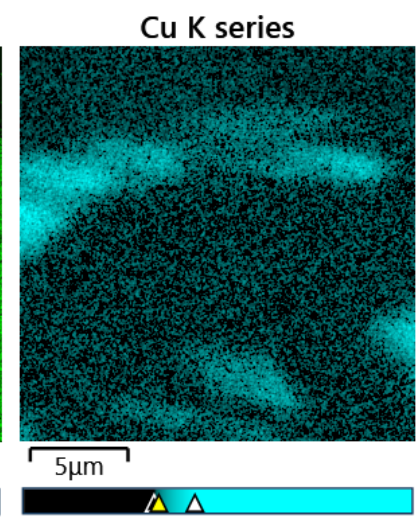

c)

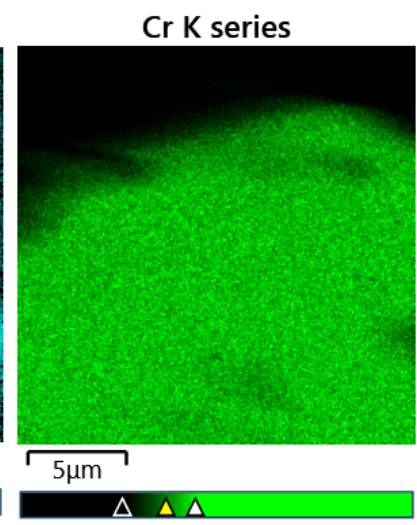

d)

Figure 7. SEM cross-section images with elemental EDXS mappinng analysis of non-modified Cu-Cr composite electrodes layer: a) and b) - cathode surface; c) and d) - anode surface. 

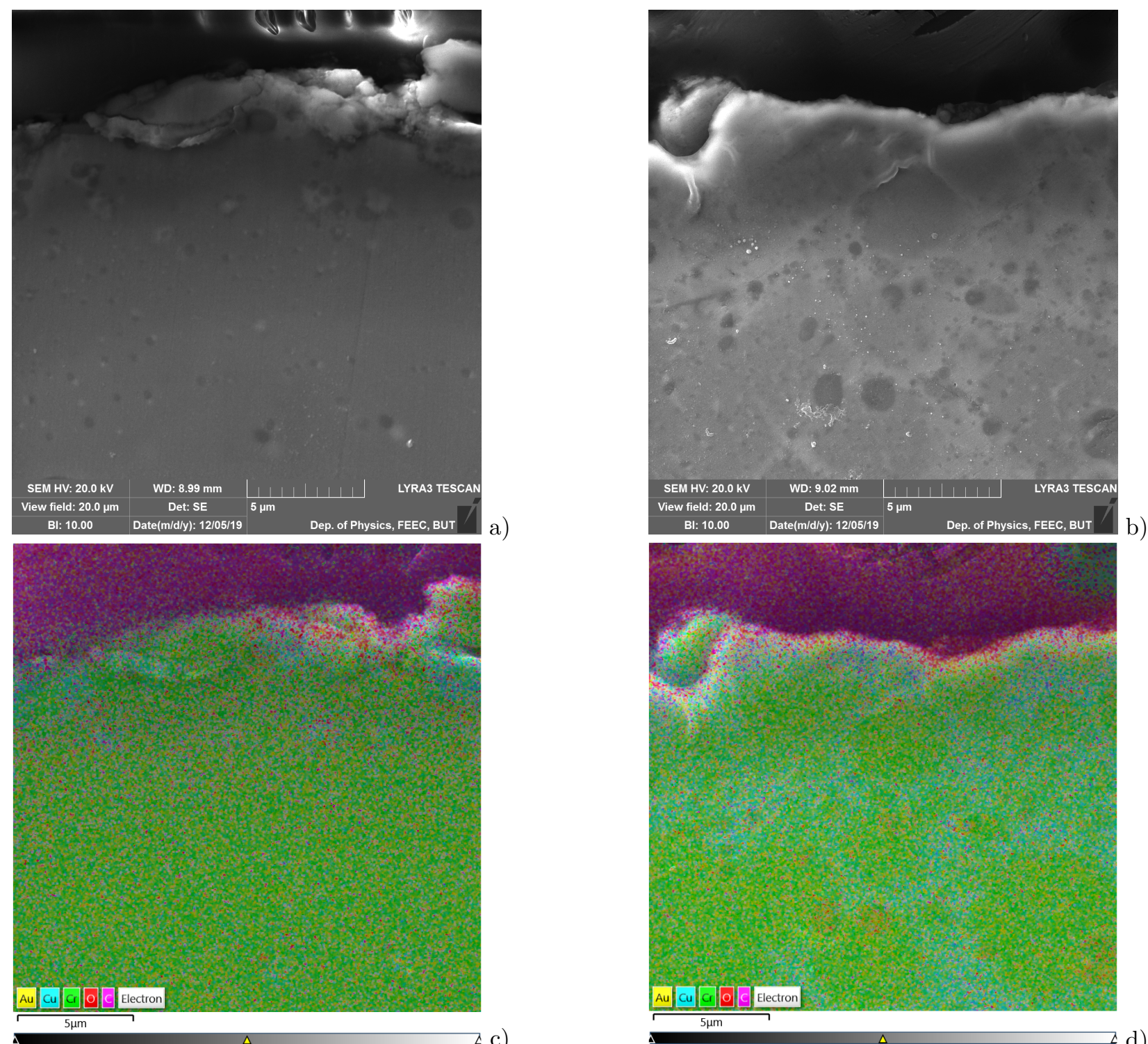

c)

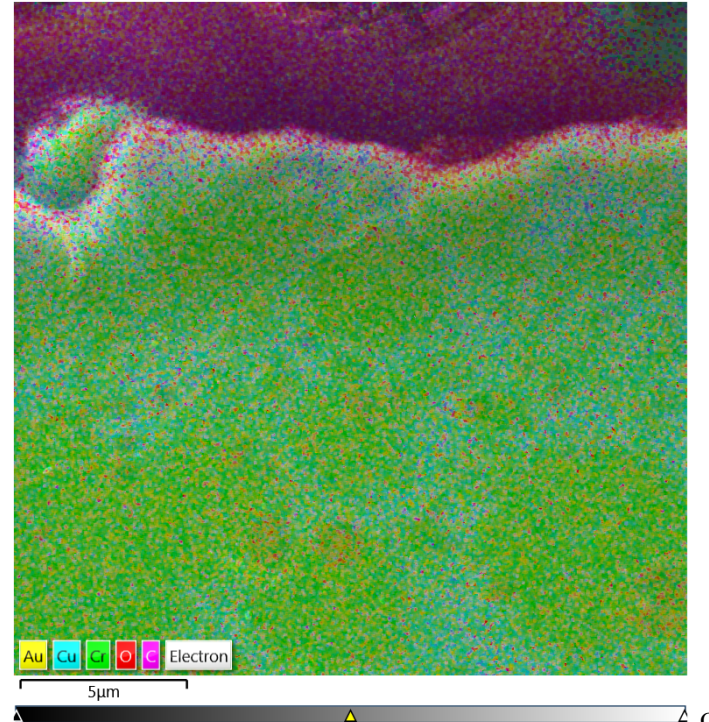

Figure 8. SEM cross-section images ( $a$ and b) and SEM cross-section images with elemental EDXS mappinng analysis ( $c$ and d) of $\mathrm{Cu}$-Cr composite electrodes layer modified by thermal action of electric arc discharge: a) and c) - cathode surface; b) and $d$ ) - anode surface.

\section{Cu K series}
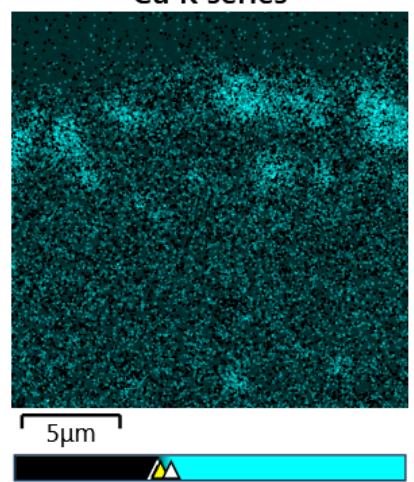

a)
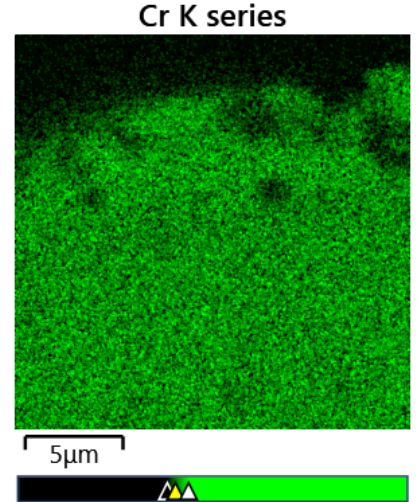

b)

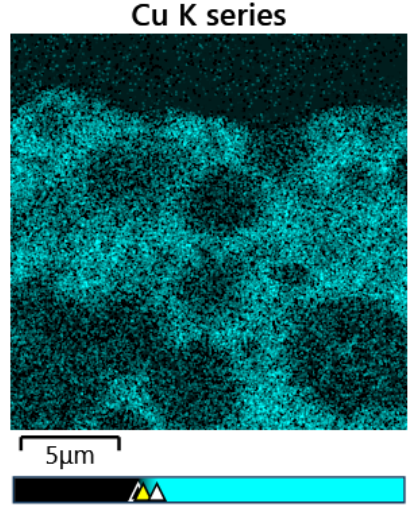

c)

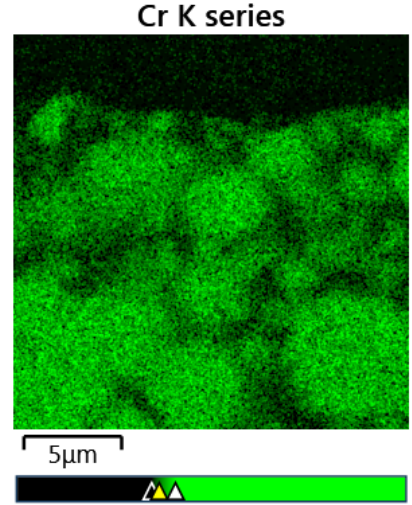

d)

Figure 9. SEM cross-section images with elemental EDXS mappinng analysis of $\mathrm{Cu}$-Cr composite electrodes layer modified by thermal action of electric arc discharge: a) and b) - cathode surface; c) and d) - anode surface. 

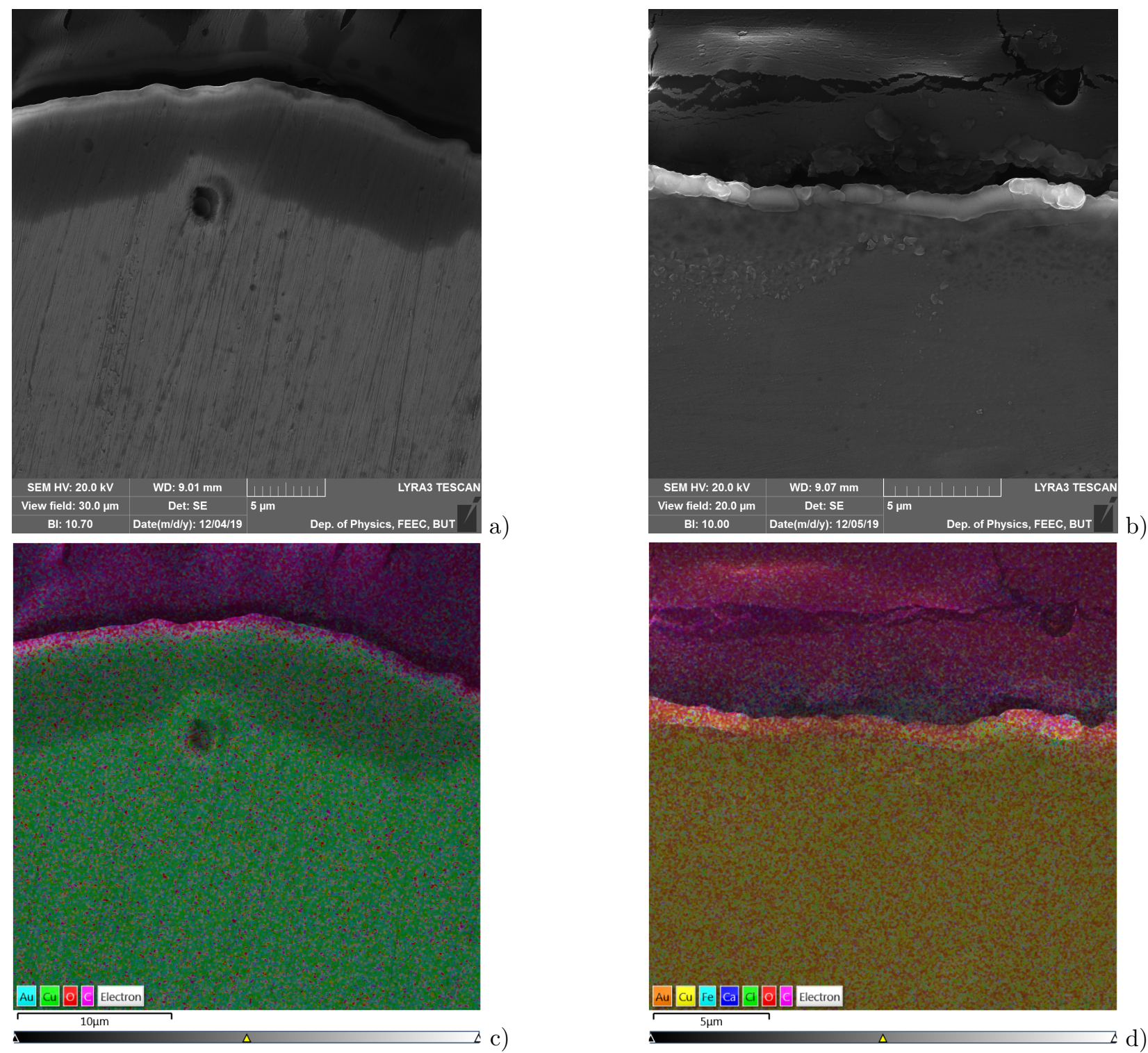

Figure 10. SEM cross-section images ( $a$ and b) and SEM cross-section images with elemental EDXS mappinng analysis ( $c$ and $d$ ) of single-component copper electrodes layer modified by thermal action of electric arc discharge: a) and c) - surface of cathode; b) and d) - of anode.

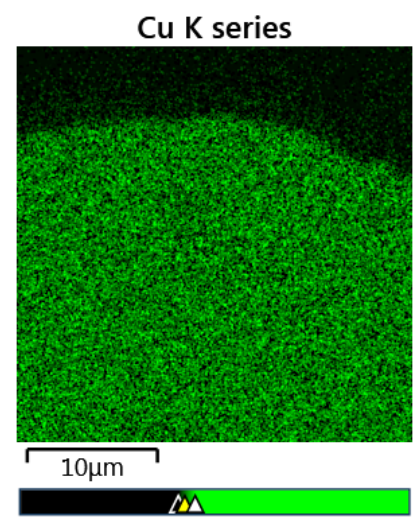

a)

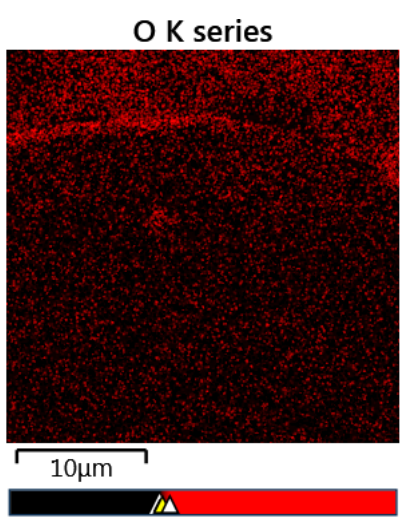

b)

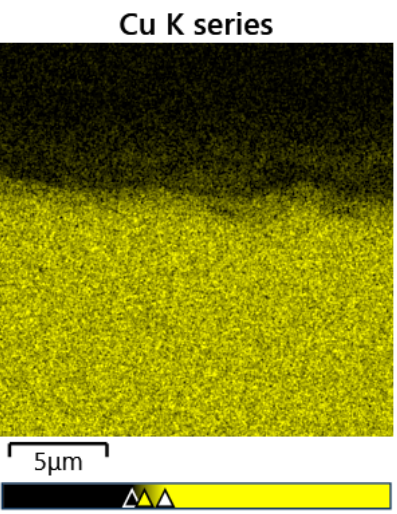

c)

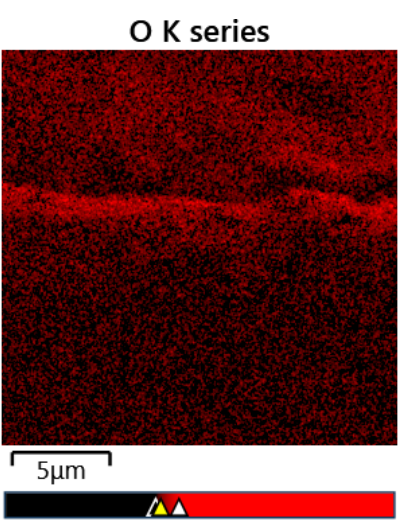

d)

Figure 11. SEM cross-section images with elemental EDXS mappinng analysis of single-component copper electrodes layer modified by thermal action of electric arc discharge: a) and b) - cathode surface; c) and d) - anode surface. 
comparison with anode in the arc energy balance.

To clarify the copper melting character in the singlecomponent copper electrodes the surface studies were carry out as well (Fig. 10). Obviously, the oxide film can be formed on electrodes working layer in condition of air atmosphere, which is confirmed by the Fig. 11 $\mathrm{b}$ and d. Moreover, the area, depleted by copper, is clearly observed in cathode working layer (Fig. 11).

\section{Conclusions}

The temporal evolution of plasma emission spectra of electric breaking arcs at current of $120 \mathrm{~A}$ in air was investigated by OES. SEM and EDXS were used to study the cross-section of working layer of electrodes surface modified by discharges heat flux.

It is concluded, that composite electrodes have better erosion resistance to arc discharge heat flux. This is confirmed by the higher emission intensities of the $\mathrm{Cu}$ I spectral lines in plasma between single-component copper electrodes in comparison with $\mathrm{Cu}-\mathrm{Cr}$ composite electrodes.

It was found, that the structure of composite electrodes is changed after influence of discharge heat flux. Specifically, the cathode surface is characterised by depleted copper area formation. This phenomenon is caused by intense evaporation from the electrode surface, modified by the thermal action of the electric arc plasma.

In the anode working layer the evaporation of the composite components is significantly less in contrast to the cathode. This can be confirmed by the presence of a larger amount of copper in the anode compared to the cathode after modifying by the heat flux from the dicharge.

In addition, the melting of both metals occurs not only in the arc spot, but also in the volume of the electrode near the working surface, which is confirmed by the blurred shapes of the components of the composite electrodes.

\section{Acknowledgements}

This work was supported from the Ministry of Education, Youth and Sports of the Czech Republic under project CZ.02.1.01/0.0/0.0/16_013/0001638 (CVVOZE Power Laboratories-Modernization of Research Infrastructure) and under joint project in the frame of research and technology collaboration No.8J19UA038 between Ukraine (No.M/90-2019) and Czech Republic (No.7AMB197UA). A. Gajdos gratefully acknowledges the Internal Grant Agency of Brno University of Technology, grant No. FEKT-S-20-6352.

\section{References}

[1] P. G. Slade. Electrical contacts, principles and applications. CRC Press, 2014.

[2] A. Papillon, J. Missiaen, J. Chaix, S. Roure, and H. Schellekens. Sintering mechanisms of $\mathrm{Cu}-\mathrm{Cr}$ metallic composites. Int. Journal of Refractory Metals and Hard
Materials, 65:9-13, 2016. doi:http:

//dx.doi.org/10.1016/j.ijrmhm.2016.11.010.

[3] B. Miao, Y. Zhang, Y. Zhao, and G. Liu. Two new $\mathrm{Cu}-\mathrm{Cr}$ alloy contact material. Proceedings IEEE 19'b Int. Symp. on Discharges and Electrical Insulation in Vacuum, pages 729-732, 2000.

[4] R. Muller. Arc-melted CuCr alloy as contact materials for vacuum intempters. Siemens Forsch.-u. Entwick1.-Ber.Bd, 17:105-111, 1988.

[5] K. Gerasimov, S. Mytnichenko, S. Pavlov, V. Chernov, and S. Nikitenko. Structural study of mechanically alloyed Cu30Cr70 by anomalous x-ray diffraction and exafs-spectroscopy. J. Alloys Compd., 252:179-183, 1997.

[6] O. Gülcan, I. Uslan, Y. Usta, and C. Çoğun. Performance and surface alloying characteristics of $\mathrm{Cu}-\mathrm{Cr}$ and $\mathrm{Cu}-\mathrm{Mo}$ powder metal tool electrodes in electrical discharge machining. Machining Science and Technology, 20(4):523-546, 2016.

doi:10.1080/10910344.2016.1191031.

[7] Z. Zhao, L. Chai, Z. Xiao, J. Tu, Y. Wang, and W. Huang. Surface modification of $\mathrm{Cu}-25 \mathrm{Cr}$ alloy induced by high current pulsed electron beam. Transactions of Nonferrous Metals Society of China, 25:1935-1943, 2015. doi:https:

//doi.org/10.1016/S1003-6326(15)63801-8.

[8] I. Lahiri and S. Bhargava. Compaction and sintering response of mechanically alloyed $\mathrm{Cu}-\mathrm{Cr}$ powder. Powder Technology, 189:433-438, 2009.

[9] D. Božić, J. Ružić, J. Stašić, and V. Rajkovic. Synthesis and characteristics of precipitation hardened $\mathrm{Cu}-\mathrm{Cr}$ alloy and multiply hardened $\mathrm{Cu}-\mathrm{Cr}-\mathrm{Al} 2 \mathrm{O} 3$ nanocomposite obtained using powder metallurgy techniques. Int. J. Mater. Res. (formerly Z. Metallkd.), 105(2):194-199, 2014.

[10] PLANSEE. Cu-Cr contacts. https : //www.plansee.com/de/produkte/komponenten/ elektrische-kontakte/kupfer-chrom-cucr.html.

[11] A. Veklich, V. Boretskij, M. Kleshych, S. Fesenko, A. Murmantsev, A. Ivanisik, O. Khomenko, O. Tolochyn, and M. Bartlova. Thermal plasma of electric arc discharge between $\mathrm{Cu}-\mathrm{Cr}$ composite electrodes. 23rd Symposium on Physics of Switching Arc, 6:27-30, 01 2019. doi:10.14311/ppt.2019.1.27.

[12] W. Rieder, M. Schussek, W. Glätzle, and E. Kny. The influence of composition and cr particle size of $\mathrm{cu} / \mathrm{cr}$ contacts onpchopping current, contact resistance, and breakdown voltage in vacuum interrupters. IEEE Transactions on components, hybrids, and manufacturing technology, 12(2):273-283, 1989. 
[13] I. Gershman, E. Gershman, A. Mironov, G. Fox-Rabinovich, and S. Veldhuis. On increased arc endurance of the $\mathrm{Cu}-\mathrm{Cr}$ system materials. Entropy, 19(386):11, 2017. doi:10.3390/e19080386.

[14] D. Heyn, M. Lindmayer, and E.-D. Wilkening. Effect of contact material on the extinction of vacuum arcs under line frequency and high frequency conditions. IEEE Transactions on components, hybrids, and manufacturing technology, 14(1):65-70, 1991.
[15] R. Methling, S. Gorchakov, M. Lisnyak, S. Franke, A. Khakpour, S. Popov, A. Batrakov, D. Uhrlandt, and $\mathrm{K}$. Weltmann. Spectroscopic investigation of a $\mathrm{Cu}-\mathrm{Cr}$ vacuum arc. IEEE Transactions on plasma science, 43(8):2303-2309, 2015.

doi:10.1109/TPS. 2015.2443856

[16] P. Atkins and L. Jones. Chemical Principles: The Quest for Insight (4th ed.). 0-7167-7355-4. W. H. Freeman and Company, 2008. 